NBER WORKING PAPER SERIES

ON BEST-RESPONSE BIDDING IN GSP AUCTIONS

\author{
Matthew Cary \\ Aparna Das \\ Benjamin Edelman \\ Ioannis Giotis \\ Kurtis Heimerl \\ Anna R. Karlin \\ Claire Mathieu \\ Michael Schwarz \\ Working Paper 13788 \\ http://www.nber.org/papers/w13788
}
NATIONAL BUREAU OF ECONOMIC RESEARCH
1050 Massachusetts Avenue
Cambridge, MA 02138
February 2008

We thank Michael Ostrovsky, David Parkes, Al Roth, and Sergei Severinov. The views expressed herein are those of the author(s) and do not necessarily reflect the views of the National Bureau of Economic Research.

NBER working papers are circulated for discussion and comment purposes. They have not been peerreviewed or been subject to the review by the NBER Board of Directors that accompanies official NBER publications.

(C) 2008 by Matthew Cary, Aparna Das, Benjamin Edelman, Ioannis Giotis, Kurtis Heimerl, Anna R. Karlin, Claire Mathieu, and Michael Schwarz. All rights reserved. Short sections of text, not to exceed two paragraphs, may be quoted without explicit permission provided that full credit, including (C) notice, is given to the source. 
On Best-Response Bidding in GSP Auctions

Matthew Cary, Aparna Das, Benjamin Edelman , Ioannis Giotis , Kurtis Heimerl, Anna R.

Karlin , Claire Mathieu , and Michael Schwarz

NBER Working Paper No. 13788

February 2008

JEL No. C15,D44,L86,M37

\section{ABSTRACT}

How should players bid in keyword auctions such as those used by Google, Yahoo! and MSN? We model ad auctions as a dynamic game of incomplete information, so we can study the convergence and robustness properties of various strategies. In particular, we consider best-response bidding strategies for a repeated auction on a single keyword, where in each round, each player chooses some optimal bid for the next round, assuming that the other players merely repeat their previous bids. We focus on a strategy we call Balanced Bidding (bb). If all players use the bb strategy, we show that bids converge to a bid vector that obtains in a complete information static model proposed by Edelman, Ostrovsky and Schwarz (2007). We prove that convergence occurs with probability 1, and we compute the expected time until convergence.

Matthew Cary

University of Washington

Seattle, WA 98195

cary@cs.washington.edu

Aparna Das

Brown University

Computer Science Department

Box 1910

Providence, RI 02912

aparna@cs.brown.edu

Benjamin Edelman

Harvard Business School

Baker Library 445

Soldiers Field

Boston, Massachusetts 02163

bedelman@fas.harvard.edu

Ioannis Giotis

University of Washington

Department of Computer Science

and Engineering

Seattle, WA 98195

giotis@cs.washington.edu
Kurtis Heimerl

University of Washington

Department of Computer Science

and Engineering

Seattle, WA 98195

heimerl@cs.washington.edu

Anna R. Karlin

University of Washington

Department of Computer Science

and Engineering

Box 352350

Seattle, WA 98195

karlin@cs.washington.edu

Claire Mathieu

Brown University

Computer Science Department

Box 1910

Providence, RI 02912

claire@cs.brown.edu

Michael Schwarz

Yahoo Research

1950 University Avenue

and NBER

mschwarz@yahoo-inc.com 


\title{
On Best-Response Bidding in GSP Auctions
}

\author{
Matthew Cary ${ }^{1}$, Aparna Das ${ }^{2}$, Benjamin Edelman ${ }^{3}$, \\ Ioannis Giotis ${ }^{1}$, Kurtis Heimerl ${ }^{1}$, Anna R. Karlin ${ }^{1}$, \\ Claire Mathieu ${ }^{2}$, and Michael Schwarz ${ }^{*}$
}

January 25, 2008

\begin{abstract}
How should players bid in keyword auctions such as those used by Google, Yahoo! and MSN? We model ad auctions as a dynamic game of incomplete information, so we can study the convergence and robustness properties of various strategies. In particular, we consider best-response bidding strategies for a repeated auction on a single keyword, where in each round, each player chooses some optimal bid for the next round, assuming that the other players merely repeat their previous bids. We focus on a strategy we call Balanced Bidding (BB). If all players use the BB strategy, we show that bids converge to a bid vector that obtains in a complete information static model proposed by Edelman et al. [4]. We prove that convergence occurs with probability 1 , and we compute the expected time until convergence.
\end{abstract}

Keywords: Keyword auctions, bidding strategies, convergence JEL classification: C72, D44

\section{Introduction}

Pay-per-click search engine advertising is an appealing approach to matching ads to users, and it is a major source of revenue for leading search engines such as Google and Yahoo!. Search engines typically show two sets of results in response to a user's search: the links the search engine has deemed relevant to the search ("organic results"), along with paid advertisements known as sponsored links. From the advertiser's perspective, this approach offers a great benefit: each advertiser can precisely target its ads based on each user's search terms. For example, if a travel agent "buys" the search term "Tahiti", then a user searching for the word "Tahiti" might be shown a link to that travel agent's

${ }^{*}$ We thank Michael Ostrovsky, David Parkes, Al Roth, and Sergei Severinov.

Author Affiliations: 1 - University of Washington, 2 - Brown University, 3 - Harvard Business School, 4 - Yahoo! Research 
web site, featuring plane tickets to Tahiti. The advertiser pays the search engine for each click on the advertiser's ad.

Keyword auctions determine which ads get assigned to which keywords and how much each advertiser pays. Advertisers choose which keywords to target and how much to bid. For each keyword of interest, an advertiser submits a bid stating the maximum amount the advertiser is willing to pay for a click. When a user searches for that keyword, an instantaneous auction determines which advertisers (among those currently bidding on that keyword) will receive advertising slots and how much each advertiser will pay for a click.

In principle, keyword engines could allocate ad slots using a multi-unit auction that causes truthful bidding, i.e. the Vickrey-Clarke-Groves ("VCG") mechanism $[14,2,6]$. Under VCG, it would be in the best interest of each participating advertiser to bid its true valuation. But in practice, no search engine uses the VCG mechanism. Rather, the most widely used keyword auction mechanism is the non-truthful Generalized Second Price ("GSP") auction described in Section 2.

Because the GSP mechanism is not truthful, participating advertisers are forced to undertake the complicated task of choosing a bidding strategy. Asdemir [1] and Edelman et al. [3] observe that standard sponsored search mechanisms can produce instability and bidding wars. Bidding strategies are particularly complex because typical search pages have room for multiple sponsored links. The position of a given sponsored link affects the chance that the link will be clicked, so the various advertising slots differ in their desirability to advertisers. Advertisers' utilities are therefore a discontinuous function of their bids. The complex bidding environment causes many advertisers to hire campaign optimization specialists to manage their bidding, often at significant cost.

Advertisers may submit updated bids whenever they like. Although these updates are often made using software robots [8], there are practical limits to how frequently an advertiser can update his bid. For example, there is a random delay between an advertiser's submission of an updated bid and the time that the updated bid is accepted by the system for use in subsequent auctions for a given keyword. For modeling purposes, we assume that exactly one randomly chosen advertiser gets to update his bid in each period, and that he knows the current bids of all other advertisers when he updates his bid.

\subsection{Our Contribution}

In this paper, we explore a natural class of best-response bidding strategies an advertiser (or his software robot) might use in a repeated keyword auction for a given search term. When an advertiser gets an opportunity to revise his bid, a natural myopic strategy is to play a best response to the current bids of other players.

The myopic best-response strategy masks substantial flexibility. Suppose that, given the last-period bids of the other bidders, some advertiser $A$ prefers

advertisement slot $s$ at price $p_{s}$ because $s$ offers the advertiser higher profit than other slots. (Other slots would either provide fewer clicks or require a higher 
payment per click.) Suppose further that the price of the (next-higher clickthrough rate) slot $s-1$ is $p_{s-1}$. The GSP mechanism allows a range of bid values that will result in the same outcome from $A$ 's perspective. (Namely, any bid between $p_{s}$ and $p_{s-1}$ will still get $A$ the same slot $s$.) What specific bid should $A$ choose? We focus on a particular choice in this range, Balanced Bidding (BB). Under $\mathrm{BB}$, the advertiser chooses his next bid $b$ so as to be indifferent between successfully winning the targeted slot $s$ at price $p_{s}$, or winning the slightly more desirable slot $s-1$ at price $b$. The dynamic system in which all players bid according to BB has a unique fixed point at a Nash equilibrium strategy profile $\mathrm{B}^{*}$ of the static game. At this point, advertisers' payments to the search engine are identical to payments under the VCG mechanism. (See Proposition 1.)

Our main result is that if a randomly chosen player updates his bid each round (according to the BB strategy), then BB bidding converges to the Nash bid vector $\mathrm{B}^{*}$ with probability 1 . (See Theorem 2.) We also estimate the average time required for convergence.

The Nash equilibria of the sponsored search static game have been understood for some time $[4,13,9]$. But it was not previously known whether there existed a natural bidding strategy in a dynamic game that would lead to these Nash equilibria. Our results show how players in a dynamic Bayesian game can use a best-response strategy to get to the equilibria of the static game of complete information.

By estimating convergence time of BB we combine the economics literature's analysis of bidding strategies with the attention to convergence time more common in computer science.

\subsection{Relation to the Literature}

Sponsored search is an intrinsically dynamic game where bidders can change their bids and learn about others' values from the history of bid changes. However, the literature has largely modeled this game as a static game. In this context, [4] and [13] independently arrived at the same equilibrium characterization of the ad auction. According to their characterization, equilibrium bids are given by the recursive formula in Equation (1). Recent papers [4, 13, 9] assume that a static model of complete information captures the essence of the dynamic environment of ad auctions. But [13] does not offer a mechanism by which the dynamic game converges to the proposed equilibrium of the static game, and [4] offers a generalized English auction that captures the dynamic nature of the game with a highly stylized ascending clock design. These papers do not not explain how the equilibria of the complete information static game might be reached in a truly dynamic incomplete information setting.

In general, characterizing equilibria of dynamic games of incomplete information is difficult or intractable. Dynamic games of incomplete information are therefore routinely modeled as simple static games. For instance, studies of price competition in airlines [10] and gasoline pricing [7] are often based on static models even though these environments are clearly not static. Little is

known about when the equilibria of static models are useful approximations of 
the equilibria of a dynamic game of incomplete information. This paper shows that in the case of ad auctions, there is a myopic best-response strategy for players of the dynamic game that converges to an equilibrium $\mathrm{B}^{*}$ of the static model.

The literature most closely related to this paper is that on best response dynamics. Best response dynamics studies the type of strategies that we consider, in which each agent plays a best response to what it encountered in the previous round (or rounds). It is well know that in wide classes of games, best response dynamics converge to a Nash Equilibrium of a one-shot game. For example, [12] shows that in "potential games," if agents play best responses to actions observed in the previous round, then the process converges to a Nash Equilibrium. However, the GSP auction is not a potential game.

Our work builds on the "weakly acyclic" notion introduced by [15] in a study of the evolution of conventions. We show that GSP is "weakly acyclic", as defined by [15], i.e., that for any starting set of player bids, there is an ordering of the players, such that letting those players in that order play a best response leads to the desired Nash equilibrium. This result is a key component of our proof of convergence.

\section{Model and Definitions}

The following model captures the essential features of sponsored search auctions. A keyword auction is defined by:

- A set of $k$ slots with click-through rates $\theta_{1}>\ldots>\theta_{k}$, where $\theta_{i}$ is the probability that the user clicks on the advertisement in slot $i$.

- A set of $n$ players (advertisers) participating in the auction, each with a private valuation $v_{i}$ for a click, $v_{1}>\ldots>v_{n}$.

- With knowledge of the mechanism and of their own private valuations, each player submits a bid to the auction. We denote player $i$ 's bid by $b_{i}$.

- The mechanism:

- computes an allocation $\pi$ of the $k$ slots to $k$ different players: $\pi_{s}$ is the identity of the player that is allocated slot $s$.

- charges a price $p_{s}$ to the player $\pi_{s}$ for each click on his advertisement.

- If player $i$ is allocated slot $s$ at price $p_{s}$, player $i$ 's expected utility is $\theta_{s}\left(v_{i}-p_{s}\right)$.

The generalized second price auction is the most common mechanism in use, including at both Google and Yahoo!.

Definition 1 The generalized second price mechanism (GSP) for keyword auctions uses the following allocation and payment rules: 
- Players are allocated slots in decreasing order of bids. ${ }^{1}$

- For each slot $s$, the payment $p_{s}$ of player $\pi_{s}$ is $b_{\pi_{s+1}}$.

Players who do not win a slot make no payment and gain no utility.

When modeled as static game of complete information, GSP has a continuum of Nash equilibria that are well understood. See $[4,13,9]$. One of these equilibria results in player payments identical to those that would be made if the mechanism being employed was VCG. This equilibrium is also attractive as it is the cheapest envy-free equilibrium from the advertisers' point of view. In that equilibrium, bids follow a recursive formula:

$$
b_{i}= \begin{cases}>b_{2} & \text { for } i=1, \\ v_{i}-\frac{\theta_{i}}{\theta_{i-1}}\left(v_{i}-b_{i+1}\right) & \text { for } 2 \leq i \leq k, \\ v_{i} & \text { for } k<i \leq n\end{cases}
$$

The highest-value bidder's bid, $b_{1}$, is unidentified because any bid $b_{1}>b_{2}$ will suffice to obtain the highest position.

We call this Nash equilibrium of GSP the VCG equilibrium of GSP, and we refer to this vector of bids as the equilibrium of the static game $\mathrm{B}^{*}{ }^{2}$

We consider a repeated keyword auction with a fixed set of $n$ players and $k$ slots. The participants in the repeated auction have the opportunity to update their bids between successive rounds. How should players bid? Naturally, a player's ultimate objective is to maximize his own expected utility over multiple rounds of the auction. However, it is difficult for one player to make predictions about the future bids - information necessary to choose an optimal bidding strategy. With limited information, a natural approach is to assume that the immediate past is the best predictor of the future. This leads to a bidding scheme where a player assumes that others' bids will remain fixed in the next round. Under this assumption, the rational choice for a player $j$ is to bid so as to win a slot $s$ that maximizes his utility $u_{j}=\theta_{s}\left(v_{j}-p_{s}\right)$. This approach offers the following definition.

\section{Definition 2 Best-Response Bidding Strategies}

$A$ best-response bidding strategy for a player $j$ is to choose a bid for the next round of a repeated keyword auction round so as to maximize his utility $u_{j}$, assuming the next-round bids of all other players $b_{-j}$ will remain fixed at their values in the previous round.

\footnotetext{
${ }^{1}$ At modern search engines, there is also a "relevance" or per-advertiser click-through rate $r_{i}$ associated with each advertiser $i$, and bidders are actually ranked (assigned slots) in decreasing order of ranking score, where the ranking score of bidder $i$ is $b_{i} r_{i}$. For notational simplicity, we assume $r_{i}=1$ for all $i$. [4] shows that our results extend to the case of arbitrary $r_{i}$ values.

${ }^{2}$ There are multiple Nash equilibria in the static game. However, [13] suggests that the equilibrium given by this formula is the most plausible, and [4] proposes a model where this equilibrium is a unique outcome.
} 
Given $b_{-j}$, denote by $p_{s}(j)$ the payment player $j$ would make if he bids so as to win slot $s$. Let $s^{*}$ be the slot the best-response bidder $j$ will target. Then if player $j$ is a best-responder, he will bid $b^{\prime} \in\left(p_{s^{*}}(j), p_{s^{*}-1}(j)\right)$. Notice that $b^{\prime}$ is not fully specified: $b^{\prime}$ is a range rather than a single point. Definition 2 therefore defines a class of strategies that are distinguished by the choice of $b^{\prime}$ within the allowed range.

What bid should player $j$ choose within the $b^{\prime}$ range? Suppose $j$ chooses a bid towards the high end of the range, thus forcing a higher price for slot $s^{*}-1$. Then the player above may retaliate by slightly underbidding $j$ - effectively swapping places with $j$. If $j$ is better off after such a retaliation, $j$ will want to force the higher player out, and the bid cannot have been an equilibrium. Thus, if bids converge to a rest point, an advertiser in a given position should not want to exchange positions with the next-higher advertiser. Conversely, if $j$ chooses a bid towards the low end of the range, the player below may respond by outbidding $j$, pushing $j$ lower. Balancing these two concerns leads to the following bidding strategy.

\section{Definition 3 Balanced Bidding}

The Balanced bidding strategy (BB) is the strategy for a player $j$ that, given $b_{-j}$,

- targets the slot $s_{j}^{*}$ which maximizes his utility, that is,

$$
s_{j}^{*}=\operatorname{argmax}_{s}\left\{\theta_{s}\left(v_{j}-p_{s}(j)\right)\right\} .
$$

- chooses his bid $b^{\prime}$ for the next round so as to satisfy the following equation:

$$
\theta_{s_{j}^{*}}\left(v_{j}-p_{s_{j}^{*}}(j)\right)=\theta_{s_{j}^{*}-1}\left(v_{j}-b^{\prime}\right) .
$$

If $s_{j}^{*}$ is the top slot, we (arbitrarily) choose $b^{\prime}=\left(v_{j}+p_{1}(j)\right) / 2$. We can thus deal with all slots uniformly by defining $\theta_{0}=2 \theta_{1}$.

The intuition behind the BB strategy is that the player bids high enough to force the prices paid by his competitors to rise. But the player does not want prices to rise so high that if one of his competitors were to just undercut him, he would regret receiving a higher slot at a price just below his own bid of $b^{\prime}$.

The following Proposition summarizes the results derived in [4]. This Proposition shows the interesting properties of BB.

Proposition 1 If all players follow the BB strategy in an auction with all distinct $\theta$ 's, then the system has a unique fixed point. At this fixed point, the revenue of the auctioneer (and the payment of each player) is equal to that of the $V C G$ equilibrium. The equilibrium bids $b_{j}^{*}$ at the fixed point satisfy the following equations:

$$
b_{j}^{*}= \begin{cases}2 b_{2} & \text { for } j=1, \\ \gamma_{j} b_{j+1}^{*}+\left(1-\gamma_{j}\right) v_{j} & \text { for } 2 \leq j \leq k, \\ v_{j} & \text { for } k<j \leq n,\end{cases}
$$

where $\gamma_{j}=\theta_{j} / \theta_{j-1}$. 


\section{Convergence Properties of the BB Strategy}

Our main result shows that when all players use the BB strategy, their payoffs converge to the payoffs they would receive in a dominant strategy equilibrium of the VCG mechanism.

Consider the update model in which, in each round, exactly one randomly chosen player updates his bid according to the BB strategy. We call this update model asynchronous bidding. Under asynchronous bidding, we show that the system converges to its fixed point. See Section 3.2.

In order to prove convergence in the asynchronous case, we must first prove convergence in the synchronous case. This building block is established in the following section.

\subsection{The Restricted Balanced Bidding Strategy and Convergence in the Synchronous Case}

Synchronous bidding is the update model in which all players see all prior-period bids, and all bidders simultaneously submit new bids for the next round. To prove convergence in this synchronous case, we examine a variant of the $\mathrm{BB}$ strategy that limits players' selection of slots. In particular, the Restricted Balanced Bidding strategy is defined as follows:

Definition 4 The Restricted Balanced Bidding (RBB) bidding strategy is the strategy for a player $j$ that, given $b_{-j}$

- targets the slot $s_{j}^{*}$ which maximizes his utility among the slots with no higher click-through rate than his current slot $s_{j}$, that is,

$$
s_{j}^{*}=\operatorname{argmax}\left\{\theta_{s}\left(v_{j}-p_{s}(j)\right): s \geq s_{j}\right\} .
$$

- chooses his bid $b^{\prime}$ for the next round so as to satisfy the following equation:

$$
\theta_{s_{j}^{*}}\left(v_{j}-p_{s_{j}^{*}}(j)\right)=\theta_{s_{j}^{*}-1}\left(v_{j}-b^{\prime}\right) .
$$

As in $\mathrm{BB}$, we define $\theta_{0}=2 \theta_{1}$ to make sure the strategy is well-defined for the first slot.

Important features of the RBB strategy are that (a) it has the same unique fixed point as BB, and (b) it provides a bidding strategy that provably converges in both the synchronous and asynchronous settings, as shown below.

The RBB restriction is crucial to the guarantee of convergence in the synchronous model. In the Appendix, we show that with three or more slots and with updates occurring synchronously, the BB strategy need not converge.

Our main RBB result is a guarantee of convergence in the synchronous case. 
Theorem 1 In the synchronous model the RBB strategy always converges to its fixed point. The number of steps until convergence is bounded by

$$
O\left(2^{k}\left(\frac{\log \left(1-\gamma^{*}\right)}{\log \gamma^{*}}+\log _{\left(1 / \gamma^{*}\right)} \frac{v_{1}}{\min _{1 \leq i \leq k}\left(v_{i}-v_{i+1}\right)}+\log _{\left(1 / \gamma^{*}\right)} \frac{\theta_{1}}{\theta_{k}}\right)\right),
$$

where $\gamma^{*}=\max _{i} \frac{\theta_{i}}{\theta_{i-1}}$. Holding click-through rates $\theta_{i}$ fixed, this simplifies to

$$
O\left(\frac{2^{k} \cdot v_{1}}{\min _{1 \leq i \leq k}\left(v_{i}-v_{i+1}\right)}\right) .
$$

Proof. We first bound the number of steps until convergence of the price of slot $k$ and the set of players who will not be allocated slots. As above, we define $\gamma_{i}=\theta_{i} / \theta_{i-1}$.

Lemma 1 Player $p$ prefers to target slot $j$ rather than slot $j-1$ if and only if

$$
\left(1-\gamma_{j}\right) v_{p}+\gamma_{j} p_{j}<p_{j-1} \text {. }
$$

Proof. From the definitions in the preceding sections, player $p$ prefers slot $j$ over slot $j-1$ if and only if:

$$
\begin{aligned}
\theta_{j}\left(v_{p}-p_{j}\right) & >\theta_{j-1}\left(v_{p}-p_{j-1}\right) \\
\theta_{j} v_{p}-\theta_{j} p_{j} & >\theta_{j-1} v_{p}-\theta_{j-1} p_{j-1} \\
v_{p}-\gamma_{j} v_{p}+\gamma_{j} p_{j} & <p_{j-1} \\
\left(1-\gamma_{j}\right) v_{p}+\gamma_{j} p_{j} & <p_{j-1}
\end{aligned}
$$

Lemma 2 At every round $t$ such that $t>t_{1}=2+\log _{\gamma^{*}}\left(\left(1-\gamma^{*}\right)\left(v_{k}-v_{k+1}\right) / v_{k+1}\right)$, where $\gamma^{*}=\max _{i>0} \theta_{i} / \theta_{i-1}$, we have:

$$
\begin{array}{ll}
b_{i}>v_{k+1} & \text { if } i \leq k, \\
b_{i}=v_{i} & \text { if } i \geq k+1 .
\end{array}
$$

Proof. Let $b$ denote the $(k+1)$ st highest bid. By definition, $b$ can never be more than $v_{k+1}$. Suppose for some round that $b$ is less than $v_{k+1}$. Take any player $i$ in $\{1,2, \ldots, k+1\}$. In the next round, $i$ will either bid his value or target some slot $j \in\{1, \ldots k\}$ and bid $b_{i}^{\prime}=\left(1-\gamma_{j}\right) v_{i}+\gamma_{j} p_{j} \geq\left(1-\gamma^{*}\right) v_{k+1}+\gamma^{*} b \geq$ $b+\left(1-\gamma^{*}\right)\left(v_{k+1}-b\right)$. In either case,

$$
\left(v_{k+1}-b_{i}^{\prime}\right) \leq \gamma^{*}\left(v_{k+1}-b\right) .
$$

Initially $v_{k+1}-b \leq v_{k+1}$. How many rounds does it take before $v_{k+1}-b<$ $\left(1-\gamma^{*}\right)\left(v_{k}-v_{k+1}\right)$ ? At most $r \leq \log _{\gamma^{*}}\left(\left(1-\gamma^{*}\right)\left(v_{k}-v_{k+1}\right) / v_{k+1}\right)$. In round $r+1$, bidders $i \in\{1, \ldots k\}$ will each bid either $v_{i}>v_{k+1}$ or bid at least

$$
\begin{aligned}
b_{i}^{\prime} & =\left(1-\gamma_{j}\right) v_{i}+\gamma_{j} p_{j} \geq\left(1-\gamma^{*}\right) v_{k}+\gamma^{*} b \\
& \geq b+\left(1-\gamma^{*}\right)\left(v_{k}-b\right)>b+\left(1-\gamma^{*}\right)\left(v_{k}-v_{k+1}\right) \\
& >v_{k+1} .
\end{aligned}
$$


In either case, their bids are above $v_{k+1}$. In round $r+2$, player $k+1$ will then bid $v_{k+1}$ while players $1,2, \ldots, k$ continue to bid above $v_{k+1}$; the other players don't get a slot, so they bid their value, and this concludes the proof.

We now need to prove that the allocation of the $k$ slots to the top $k$ players converges to a fixed point. At any time, for any $i \in[1, k]$, consider the players allocated slots $[i+1, k]$. They are called stable if their bids and prices satisfy Equation (2), that is, the allocation is in order of decreasing values, and if $\pi(j)$ is the player currently allocated slot $j$, then the last bids of those players satisfy

$$
b_{\pi(j)}=\gamma_{j} b_{\pi(j+1)}+\left(1-\gamma_{j}\right) v_{\pi(j)},
$$

for every $j \in[i+1, k]$. If the players allocated all slots $[1, k]$ form a stable set, then we have reached the fixed point of the RBB strategy.

Assume that the current setting is not (yet) a fixed point of the RBB strategy. Let $A$ be the maximum stable set, with associated $i \geq 2$, and let $B$ be the set of players in slots $[1, i]$. Let $b_{\min }$ denote the minimum bid from players of $B$. We define a partial order over sets of players. We say that $A^{\prime} \succ A$ if either $A \subset A^{\prime}$ and $A \neq A^{\prime}$, or if the player of minimum value in the symmetric difference of $A$ and $A^{\prime}$ belongs to $A^{\prime}{ }^{3}$ This will be our measure of progress. In the next round, observe that, following the RBB strategy, players in $A$ still bid in the same way as before. Let $b_{\min }^{\prime}$ be the new minimum bid from players of $B$, and $p$ be the player of $B$ whose bid is $b_{\min }^{\prime}$. There are three cases to consider.

1. $p$ bids below $p_{i}$. Let $j \in[i+1, k]$ be the slot which was targeted by $p$. Because $p$ targeted slot $j, p$ prefers slot $j$ to slot $j-1$, and so, by Lemma 1 , the bid of $p$ is less than $p_{j-1}$. By the definition of RBB the bid is $\left(1-\gamma_{j}\right) v_{p}+\gamma_{j} p_{j}>p_{j}$, which falls in the interval $\left(p_{j-1}, p_{j}\right)$, so $p$ will indeed be allocated slot $j$.

Recall that $\pi(j) \in A$ denotes the player who was in slot $j$ in the previous round. Since set $A$ is stable, the definition of RBB requires $p_{j-1}=\left(1-\gamma_{j}\right) v_{\pi(j)}+\gamma_{j} p_{j}$. This is greater than the bid of $p$, so it follows that $v_{\pi(j)}>v_{p}$. Moreover, since $p$ prefers slot $j$ to slot $j+1$, again by Lemma 1, we must have $\left(1-\gamma_{j+1}\right) v_{p}+\gamma_{j+1} p_{j+1}>p_{j}=\left(1-\gamma_{j+1}\right) v_{\pi(j+1)}+$ $\gamma_{j+1} p_{j+1}$, and so $v_{p}>v_{\pi(j+1)}$. Thus $A^{\prime}=\left\{p^{\prime} \in A: v_{p^{\prime}}<v_{p}\right\} \cup\{p\}$ is a stable set, and $A^{\prime} \succ A$.

2. $p$ targeted slot $i$. Then $p$ is allocated slot $i, A^{\prime}=A \cup\{p\}$ is a stable set, and $A^{\prime} \succ A$.

3. $p$ targeted some slot $j \leq i-1$. Then $A$ is still a stable set, and $b_{\min }=p_{i-1}$ has increased: $b_{\min }^{\prime}=\left(1-\gamma_{j}\right) v_{p}+\gamma_{j} p_{j} \geq b_{\min }+\left(1-\gamma^{*}\right)\left(v_{p}-b_{\min }\right)$.

We now define a new variable $x$. We will prove that Case 3 can only happen a bounded number $x$ of times (where $x$ depends on the $\theta_{j}$ 's and the $v_{j}$ 's but not on the bids) before Case 1 or 2 must occur.

\footnotetext{
${ }^{3}$ This corresponds to a lexicographic ordering of the stable sets.
} 
Definition 5 Let $x=\log _{1 / \gamma^{*}}\left(\left(v_{1}-v_{k+1}\right) / \varepsilon\right)$ with $\varepsilon$ as defined in Lemma 3.

Thus, the maximum stable set must change at least once every $x$ rounds. When the maximum stable set changes, it is replaced by a set which is larger in the $\succ$ ordering. This implies that the system converges to a fixed point and that the number of rounds until convergence is bounded by $2^{k}(x+1)$, hence the Theorem. First, a useful technical lemma.

Lemma 3 Let $\varepsilon=(1 / 2) \theta_{k}\left(1-\gamma^{*}\right) \min _{q \neq q^{\prime}}\left|v_{q}-v_{q^{\prime}}\right| / \theta_{1}$. If $p_{i-1}>v_{p}-\varepsilon$ and $v_{p}>p_{i}$, then player $p$ prefers slot $i$ to any slot $j<i$.

Proof. From player $p$ 's viewpoint, the utility of slot $i$ is $\theta_{i}\left(v_{p}-p_{i}\right)$, the utility of slot $j<i$ is $\theta_{j}\left(v_{p}-p_{j}\right)<\theta_{j}\left(v_{p}-p_{i-1}\right)$, and the ratio is

$$
\frac{\theta_{j}\left(v_{p}-p_{i-1}\right)}{\theta_{i}\left(v_{p}-p_{i}\right)} \leq \varepsilon \frac{\theta_{j}}{\theta_{i}\left(v_{p}-p_{i}\right)} \leq \varepsilon \frac{\theta_{1}}{\theta_{k}\left(v_{p}-p_{i}\right)} .
$$

Now,

$$
\begin{aligned}
v_{p}-p_{i} & =v_{p}-\left(\left(1-\gamma_{i+1}\right) v_{\pi(i+1)}+\gamma_{i+1} p_{i+1}\right) \\
& =\left(1-\gamma_{i+1}\right)\left(v_{p}-v_{\pi(i+1)}\right)+\gamma_{i+1}\left(v_{p}-p_{i+1}\right),
\end{aligned}
$$

which is at least $\left(1-\gamma^{*}\right) \min _{q \neq q^{\prime}}\left|v_{q}-v_{q^{\prime}}\right|$. Plugging this into the previous expression proves the Lemma.

We now return to proving that Case 3 happens for at most $x$ consecutive rounds. Assume that Case 3 happens for $x$ consecutive rounds. Let $p_{\min }$ be the player in $B$ whose value is minimum, and let $v_{\min }$ be its value. Let $b_{\min }^{(t)}$ be the minimum bid of players in $B$ after $t$ rounds, $0 \leq t \leq x$. If $p \in B$ is the player defining the minimum bid in round $t+1$, we have:

$$
b_{\min }^{(t+1)} \geq\left(1-\gamma^{*}\right) v_{p}+\gamma^{*} b_{\min }^{(t)} \geq\left(1-\gamma^{*}\right) v_{\min }+\gamma^{*} b_{\min }^{(t)} .
$$

After $x$ rounds, we get $b_{\min }^{(x)} \geq v_{\min }-\left(\gamma^{*}\right)^{x}\left(v_{\min }-b_{\min }^{(0)}\right)$, hence $b_{\min }^{(x)} \geq v_{\min }-$ $\left(\gamma^{*}\right)^{x}\left(v_{i}-v_{k+1}\right)$. Plugging in the value of $x$ yields $b_{\min }^{(x)} \geq v_{\min }-\varepsilon$. From Lemma 3 , we know that $p_{\min }$ prefers slot $i$ to any slot $j<i$. In the next round $p_{\text {min }}$ targets slot $i$ and has to be the minimum bidder from $B$, so we leave Case 3 and enter Case 2. Thus there are at most $x$ occurrences of Case 3 between any two occurrences of Case 1 or Case 2, and the Theorem is proved.

\subsection{Convergence of the BB Strategy in the Asynchronous Case}

We now turn to our main result, convergence of the BB strategy in the asynchronous case - where each round gives a single random bidder the opportunity to update his bid according to BB. 
Theorem 2 In the asynchronous model where players bid in random order, the system converges to its fixed point with probability 1. Convergence occurs in $O\left(t_{1}(n \log k)+n \log n+k^{t_{2}}\right)$ steps on average. Here $t_{1}$ is defined as in Lemma 2, $t_{2}=2^{k}(1+x)$, and $x$ is as defined in Definition 5 .

Proof. At time $t$, a player $p$ is activated if $p$ is the player who updates his bid (while others repeat their previous bids). To prove convergence in the asynchronous case, we revisit each step of the proof of Theorem 1 (showing convergence in the synchronous case). To bound convergence time, we analyze the expected number of asynchronous activations required to achieve each step of the synchronous proof, given that the active player is selected uniformly in each asynchronous round.

Stage one. Following Theorem 1, we must first assure that $b_{i}>v_{k+1}$ for $i \leq k$, and $b_{i}=v_{i}$ for $i \geq k+1$. Lemma 2 showed this step could be obtained in $t_{1}$ rounds in the synchronous setting. To use Lemma 2 in the asynchronous case, we must activate each of the top $k+1$ players once per $t_{1}$ rounds. Because the active player is picked uniformly out of $n$ players, in expectation $O(n \log (k))$ activations are required per round to ensure that all top $k$ players update their bids. Thus, after an average of $O\left(t_{1} n \log (k)\right)$ activations, the top $k+1$ players will all be bidding as required to complete stage one. Now each losing player must be activated once so that each losing player bids its value. To ensure that all the $n-(k+1)$ losing players are activated at least once will require an additional $O(n \log (n-k))$ activations. Thus in total the expected number of activations to complete stage one in the asynchronous setting is $O\left(t_{1} n \log (k)+n \log (n)\right)$.

Stage two. Continuing in the structure of Theorem 1, we must assure that the top $k$ players are sorted correctly. Progress entails increasingly better stable sets (where "better" is defined by the lexicographic ordering of Theorem 1).

After stage one, no losing player can afford a slot, so we can ignore all losing players. If a losing player is activated, he bids his value which must be less than $b_{k}$ (because he is a losing player). Thus the bids from losing players will not interfere with the convergence of the top $k$ players. However, losing players can still consume activations. We therefore later add a budget of steps to cover the expected number of player activations required for losing players.

We now define a sequence $T$ of activations of the top $k$ bidders to complete stage two. The sequence $T$ will be partitioned into phases, corresponding to stable sets (defined in the proof of Theorem 1). Let $A$ be the current stable set, $[i+1, k]$ be the slots occupied by the players of $A$, and $B$ be the set of players occupying slots $[1, i]$. Let $p_{\min }$ be the player in $B$ whose value is minimum. Consider the three cases enumerated in the proof of Theorem 1 . The occurrence of Case 1 or Case 2 always results in a new stable set. For each stable set, Case 3 can occur at most $x$ times, after which a bid from $p_{\min }$ is guaranteed to result in a new stable set.

To achieve these results in the asynchronous case, we repeatedly activate the player in slot $i$ until either Case 1 or Case 2 occurs. If neither case occurs after $x$ activations, then we activate player $p_{\min }$. At this point a new stable set is 
formed, the current phase ends, and we move on to the sequence of activations required by the next stable set. This completes the definition of sequence $T$. As there are $2^{k}$ stable sets and each stable set requires at most $x+1$ activations, the length $T$ is bounded by $t_{2}=2^{k}(x+1)$.

How long will it take to activate players in the order given by sequence $T$ ? With $k$ players, the probability of activating the correct player is at least $1 / k$ per step. (Recall that we excluded losers.) Thus the correct sequence of activations will occur after about $k^{t_{2}}$ steps.

Finally, we add a set of steps for losing players. We expect $(1-k / n) k^{t_{2}}$ losing player activations to occur prior to convergence. The second stage therefore requires expected total activations of $k^{t_{2}}+(1-k / n) k^{t_{2}}=O\left(k^{t_{2}}\right)$.

Summation. Adding the expected steps required in stage one to those from stage two, convergence is reached on average in $O\left(t_{1}(n \log k)+n \log n+k^{t_{2}}\right)$ steps. In any event, convergence occurs with probability 1 after a finite time. (See [11].) This proves convergence, and the Theorem is complete.

Our bound on the convergence time of BB appears to be quite loose and is a worse case bound. Consider convergence speed if the number of positions, $k$, and the click-through rates, $\theta_{i}$, are held constant as the number of bidders, $n$, increases. Substituting $t_{1}$ and $x$ into Theorem 2 and simplifying, the expected number of rounds until convergence is in the order of

$n \log k \log _{1 / \gamma^{*}} \frac{v_{k}}{\left(v_{k}-v_{k+1}\right)\left(1-\gamma^{*}\right)}+\left(\frac{v_{1} \theta_{1}}{\min _{1 \leq i \leq k}\left(v_{i}-v_{i+1}\right)\left(1-\gamma^{*}\right) \theta_{k}}\right)^{2^{k} / \log \left(1 / \gamma^{*}\right)}$

where $\gamma^{*}=\max _{i} \frac{\theta_{i}}{\theta_{i-1}}$. Notice that $n$ appears only at the start of the expression. So the convergence time is linear in the number of bidders, $n$.

We have run simulations to study the speed of convergence in practice. We have observed that convergence is significantly faster than Theorem 2 indicates - no more than a polynomial in $n$, the number of bidders.

Figure 1 shows convergence speed as more bidders join the auction and as more slots are added (both occurring at the same rate). The speed of convergence, measured in number of bids per bidder, appears to increase slowly in the number of bidders.

Figure 2 shows convergence behavior in one set of simulations. In this simulation, ten bidders asynchronously updated their bids in random order, all using the BB strategy. After 329 updates, all bids had reached the B* equilibrium. This outcome is typical of our simulated bidding outcomes.

\section{Conclusions}

Keyword auctions have become a critical source of revenue for companies such as Google and Yahoo!, among others. This new form of advertising has provided a new way for advertisers to reach customers. But advertisers also face the complex task of optimizing bids to increase their exposure while avoiding unnecessary costs. 


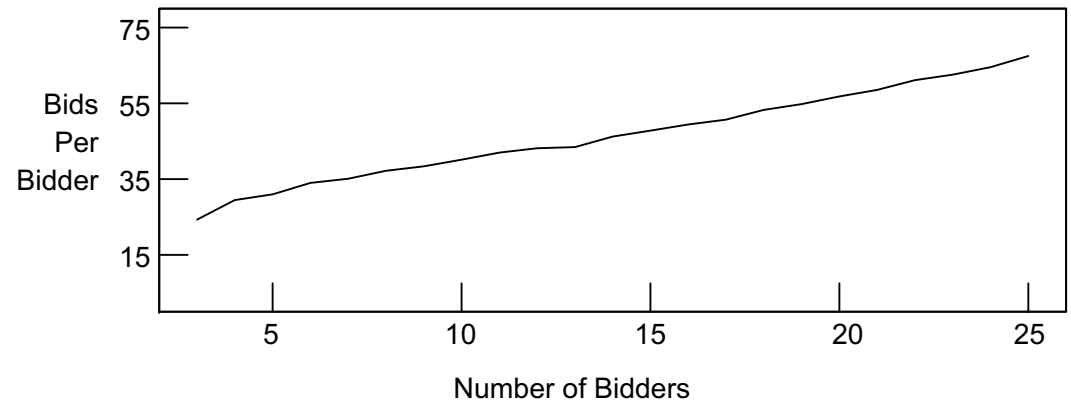

Figure 1: Convergence Speed as a Function of Number of Bidders

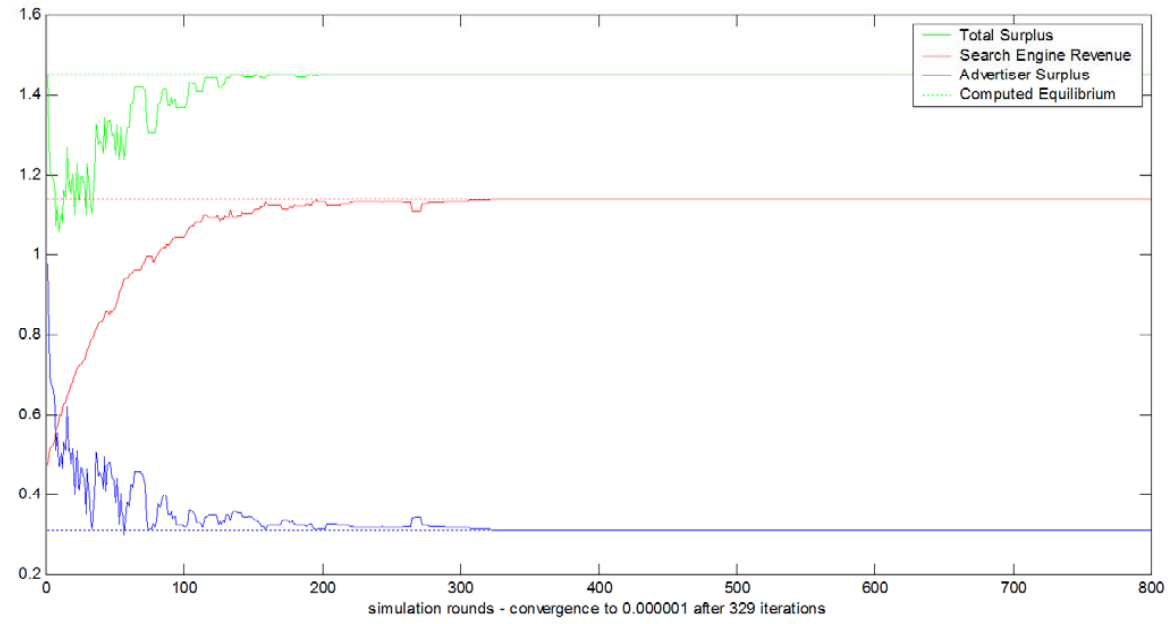

Figure 2: Simulated Convergence Profile of BB

Our analysis considers a natural class of bidding strategies inspired by equilibrium analysis and the notion of envy-freeness introduced in [4]. These bidding strategies attempt to increase advertiser utility under limited assumptions about other players' behavior. We have shown that if all players follow the BB strategy, bids are certain to converge to a Nash equilibrium of the static game. We have further determined the expected time until convergence. Finally, we have noted the desirable properties this equilibrium offers to advertisers - including envy-freeness, lowest cost to advertisers (among all Nash equilibria of the static game of complete information), and feasible implementation based on information available to advertisers. 


\section{Acknowledgements}

This research was supported in part by NSF grant CCF-0635147, a Yahoo Research Alliance gift to Anna Karlin, and the Division of Research and Faculty Development at Harvard Business School. 


\section{References}

[1] K. Asdemir, Bidding patterns in search engine auctions, in: Second Workshop on Sponsored Search Auctions, ACM Electronic Commerce, 2006.

[2] E. H. Clarke, Multipart pricing of public goods, Public Choice 11 (1971) $17-33$.

[3] B. Edelman, M. Ostrovsky, Strategic bidder behavior in sponsored search auctions, Decision Support Systems (2007) 192-198.

[4] B. Edelman, M. Ostrovsky, M. Schwarz, Internet advertising and the generalized second price auction: Selling billions of dollars worth of keywords, American Economic Review (2007) 242-259.

[5] J. Feng, H. K. Bhargava, P. D.M., Implementing sponsored search in web search engines: Computational evaluation of alternative mechanisms., INFORMS Journal on ComputingForthcoming.

[6] T. Groves, Incentives in teams, Econometrica 41 (1973) 617-631.

[7] J. Houde, Entry and exit in a price regulated industry: Gasoline retailing in quebec, working paper (2007).

[8] B. Kitts, B. Leblanc, Optimal bidding on keyword auctions, Electronic Markets (2004) 186-201.

[9] S. Lahaie, An analysis of alternative slot auction designs for sponsored search, in: EC '06: Proceedings of the 7th ACM conference on Electronic commerce, ACM Press, New York, NY, USA, 2006.

[10] R. McAfee, V. te Velde, Dynamic pricing in the airline industry, to appear in Production and Operations Management, Special Issue on Revenue Management and Dynamic Pricing (2007).

[11] M. Mitzenmacher, E. Upfal, Probability and Computing: Randomized Algorithms and Probabilistic Analysis, Cambridge University Press, 2005.

[12] D. Monderer, L. Shapley, Potential games, Games and Economic Behavior 14(1) (1996) 124-143.

[13] H. Varian, Position auctions, International Journal of Industrial Organization.

[14] W. Vickrey, Counterspeculation, auctions and competitive sealed tenders, Journal of Finance (1961) 8-37.

[15] H. Young, The evolution of conventions, Econometrica 61(1) (1993) 57-84. 


\section{Appendix: Convergence Properties of the Synchronous BB Strategy}

The body of the paper analyzes bidding sequences that are restricted synchronously, or asynchronously in random order. But what if bidders bid synchronously without restriction, or bid asynchronously in a predetermined order? In general, synchronous bidding and predetermined bid order can produce cycling. ${ }^{4}$

The following theorem characterizes outcomes in synchronous environments and in environments where bidding order is predetermined.

Theorem 3 Consider a repeated keyword auction where all players are using the $\mathrm{BB}$ strategy starting with arbitrary initial bids. We have:

1. A 2-slot auction system always converges to its fixed point in both the synchronous and asynchronous models. The number of rounds until convergence in the synchronous model is $O\left(\log \left(\left(v_{2}-v_{3}\right) / v_{3}\right)\right)$, where the constant depends on the click-through rates $\theta_{1}$ and $\theta_{2}$.

2. There exists a 3-slot auction system and set of initial bids which does not converge in the synchronous model.

3. There exists a 3-slot auction system, a set of initial bids, and an order in which the players update which does not converge in the asynchronous model with a predetermined order of updates.

\section{Proof of Part 1:}

Lemma 4 At every round $t$ such that $t>t_{1}=2+\log _{\gamma^{*}}\left(\left(1-\gamma^{*}\right)\left(v_{2}-v_{3}\right) / v_{3}\right)$, where $\gamma^{*}=\max \left\{\theta_{1} / \theta_{0}, \theta_{2} / \theta_{1}\right\}$, we have:

$$
\begin{aligned}
b_{1}, b_{2} & >v_{3}, \\
b_{3} & =v_{3} .
\end{aligned}
$$

Proof. Let $b$ denote the third highest bid. By the second round, $b$ can never be more than $v_{3}$. Suppose for some round that $b$ is less than $v_{3}$. Take a player $i$ in $\{1,2,3\}$. In the next round, $i$ will bid his value or target some slot $j \in\{1,2\}$ and bid $b_{i}^{\prime}=\left(1-\gamma_{j}\right) v_{i}+\gamma_{j} p_{j} \geq\left(1-\gamma^{*}\right) v_{3}+\gamma^{*} b=b+\left(1-\gamma^{*}\right)\left(v_{3}-b\right)$. In either case,

$$
\left(v_{3}-b_{i}^{\prime}\right) \leq \gamma^{*}\left(v_{3}-b\right) .
$$

Initially $v_{3}-b \leq v_{3}$. It takes at at most $r=\log _{\gamma^{*}}\left(\left(1-\gamma^{*}\right)\left(v_{2}-v_{3}\right) / v_{3}\right)$ before $v_{3}-b<\left(1-\gamma^{*}\right)\left(v_{2}-v_{3}\right)$. (Recall that $\gamma^{*}<1$ as the $\theta_{i}$ 's are decreasing.)

\footnotetext{
${ }^{4}$ Consider a simultaneous-move "battle of the sexes" game.
} 
In round $r+1$, bidders $i \in\{1,2\}$ will each bid either $v_{i}>v_{3}$ or target a slot $j \in\{1,2\}$ and bid

$$
\begin{aligned}
b_{i}^{\prime} & =\left(1-\gamma_{j}\right) v_{i}+\gamma_{j} p_{j} \geq\left(1-\gamma^{*}\right) v_{2}+\gamma^{*} b \\
& =b+\left(1-\gamma^{*}\right)\left(v_{2}-b\right)>b+\left(1-\gamma^{*}\right)\left(v_{2}-v_{3}\right) \\
& >v_{3}
\end{aligned}
$$

hence in either case their bids are both above $v_{3}$. In round $r+2$, player 3 will then bid $v_{3}$ while players 1 and 2 keep on bidding above $v_{3}$; this concludes the proof of the lemma.

Once the conditions of Lemma 4 hold, the price of slot 2 is fixed at $p_{2}=v_{3}$. Let $T_{2}=b_{2}^{*}=\left(1-\theta_{2} / \theta_{1}\right) v_{2}+\left(\theta_{2} / \theta_{1}\right) p_{2}$ and $T_{1}=\left(1-\theta_{2} / \theta_{1}\right) v_{1}+\left(\theta_{2} / \theta_{1}\right) p_{2}$. If the last bids of players 1 and 2 were $b_{1}$ and $b_{2}$ then, in the next round, their bids are:

$$
\begin{aligned}
& b_{1}^{\prime}= \begin{cases}T_{1} & \text { if } b_{2}>T_{1}, \\
\left(v_{1}+b_{2}\right) / 2 & \text { otherwise. }\end{cases} \\
& b_{2}^{\prime}= \begin{cases}T_{2} & \text { if } b_{1}>T_{2}, \\
\left(v_{2}+b_{1}\right) / 2 & \text { otherwise. }\end{cases}
\end{aligned}
$$

Let $b_{\min }=\min \left(b_{1}, b_{2}\right)$ be the minimum of the two bids.

Lemma 5 After at most $t_{2}$ rounds, we have $b_{\min } \geq T_{2}$, where

$$
t_{2} \leq t_{1}+2 \frac{\theta_{1}-\theta_{2}}{\theta_{2}} .
$$

Proof. Assume that at time $t>t_{1}$, we have $b_{\min }<T_{2}$. Then it is easy to check that at the next round we have $b_{\min }^{\prime} \geq\left(v_{2}+b_{\min }\right) / 2$. This implies

$$
b_{\text {min }}^{\prime}-b_{\min } \geq \frac{v_{2}-b_{\min }}{2} \geq \frac{v_{2}-T_{2}}{2}=\frac{\theta_{2}}{\theta_{1}} \frac{v_{2}-p_{2}}{2}=\delta .
$$

Since at time $t_{1}$ we have $b_{\min } \geq v_{3}$, we will reach $b_{\min }^{\prime} \geq T_{2}$ after an additional number of rounds bounded by $\left(T_{2}-v_{3}\right) / \delta$.

Finally, since at round $t_{2}$ we have $b_{\min } \geq T_{2}$, at time $t_{2}+1$ we will have $b_{2}^{\prime}=T_{2}<T_{1}$, and therefore at time $t_{2}+2$ we will have $b_{2}^{\prime \prime}=T_{2}=b_{2}^{*}$ and $b_{1}^{\prime \prime}=\left(v_{1}+T_{2}\right) / 2=b_{1}^{*}$; we have reached equilibrium. This proves Part 1 of the Theorem in the synchronous model.

Proof of Part 2: If there are more than two slots, convergence is not guaranteed even when all players follow the BB strategy. Consider the following counterexample.

Let there be three slots with $\theta_{1}=1, \theta_{2}=2 / 3, \theta_{3}=1 / 3$ and four players with values 161, 160, 159 and 100. Let the initial bids of 130.5, 130, 129.5 and 100, respectively. Then the bidding evolves as shown below. Notice that the bids cycle but do not converge. 


\begin{tabular}{|cc|ccc|}
\hline \multicolumn{2}{|c|}{ Bidder } & \multicolumn{3}{c|}{ Round } \\
ID & Value & 1 & 2 & 3 \\
\hline A & 161 & 130.5 & 145.5 & 130.5 \\
B & 160 & 130 & 145.25 & 130 \\
C & 159 & 129.5 & 144.75 & 129.5 \\
D & 100 & 100 & 100 & 100 \\
\hline
\end{tabular}

Consider the dynamics of this counterexample. Bidder D has the lowest value and is inactive; at any of the prices listed, he cannot receive a slot because the prices are all above his utility. Meanwhile, bidders A, B, and C cyclically compete for the available slots. It the first round, $\mathrm{A}, \mathrm{B}$, and $\mathrm{C}$ all target the lowest slot. Bidder A bids low enough that, in the next round, all three choose to target the first slot. Seeing these high bids, the anticipated price for the top slot is too high when compared with the low price anticipated for the last slot. Thus for the third round, bidders A, B, and C again target the last slot yielding the same bids as in the first round. Round four will match round two, and the cycle will continue forever.

In this example the click-through rates follow a simple geometric sequence, similar to those observed in practice [5]. Note that in this example the bids are well-behaved, in the sense that the bids are in the same order as the players' values. Hence such regularity is not sufficient for convergence. Finally, even though we used our convention of $\theta_{0}=2$, a similar example can be constructed where the players are cycling while targeting intermediate slots. This concludes the proof of part 2.

Proof of Part 3: Consider the following three slot example with $\theta_{1}=1$, $\theta_{2}=0.1$ and $\theta_{3}=0.09$. Let the top three bidders place bids in predetermined order given by their lexicographic ordering (left-most column). Notice the cycling that results: bids in round 7 exactly match those from round 1 , and the cycle will continue forever.

\begin{tabular}{|cc|ccccccc|}
\hline \multicolumn{2}{|c|}{ Bidder } & \multicolumn{7}{|c|}{ Round } \\
ID & Value & 1 & 2 & 3 & 4 & 5 & 6 & 7 \\
\hline A & 102 & 19.2 & 80.8 & 80.8 & 80.8 & 19.2 & 19.2 & 19.2 \\
B & 101 & 19.1 & 19.1 & 90.9 & 90.9 & 90.9 & 19.1 & 19.1 \\
C & 100 & 59.6 & 59.6 & 59.6 & 95.45 & 95.45 & 95.45 & 59.6 \\
D & 10 & 10 & 10 & 10 & 10 & 10 & 10 & 10 \\
\hline
\end{tabular}

